\title{
Polymer Composite Manufacturing by FDM 3D Printing Technology
}

\author{
Katarzyna Bryll ${ }^{1, a}$, Elżbieta Piesowicz ${ }^{2}$, Paweł Szymański ${ }^{3}$, Wojciech Ślączka ${ }^{4}$ and Marek Pijanowski ${ }^{1}$ \\ ${ }^{1}$ Institute of Basic Technical Sciences, Faculty of Marine Engineering, Maritime University of Szczecin, ul. Waly \\ Chrobrego 1-2, 70-500 Szczecin, Poland \\ ${ }^{2}$ Institute of Materials Science and Engineering, Faculty of Mechanical Engineering and Mechatronics, West \\ Pomeranian University of Technology, Al. Piastów 19, 70-310 Szczecin, Poland. \\ ${ }^{3}$ Institute of Materials Technology, Faculty of of Machine Construction and Management, Poznan University of \\ Technology ul. Piotrowo 3, 60-965 Poznan, \\ ${ }^{4}$ Maritime Risk Analisis Center, , Faculty of Navigation, Maritime University of Szczecin ul. Waly Chrobrego 1-2, 70- \\ 500 Szczecin, Poland
}

\begin{abstract}
D printing technology was developed nearly 30 years ago. One of its characteristics is that instead of removing materials, 3D printing creates $3 \mathrm{D}$ elements directly from $\mathrm{CAD}$ models, adding one layer of material on another. This offers a beneficial capability of making complex elements in terms of shape and materials, impossible to be manufactured by traditional methods. Owing to intensive research in recent years, considerable progress has been achieved in the development and commercialisation of new innovative processes of 3D printing by fused deposition modeling (FDM), including printing of composite materials. The study outlines the main methods of creating polymer composite structures using FDM technology.
\end{abstract}

\section{Introduction}

The idea of 3D printing has been known for decades [1]. Additive manufacturing is today used for making tools and rapid prototypes at the design and construction stage in aviation, automobile and military industries, architecture, medicine, goldsmithing, food and footwear industries, where in a relatively short time a real model can be made to be presented, apart from printed documentation, to a customer or sponsor. The development of 3D printing technology has enabled making prototypes as well as spare parts or complete devices with attractive properties, usually of small size. 3D printers in medicine are used for printing prosthetics, implants, and bioprints using cells (wound healing) [1-5].

Extended applicability and wider dissemination of 3D printing, as well as higher performance requirements necessitate seeking multimaterial solutions and use of state-of-the-art composites [2-9].

Composite properties depend on many factors, i.e. properties of component phases, their relative quantities and geometry (particle size, shape, orientation and distribution of the reinforcement phase in the matrix) as well as the strength of interfacial boundaries or production methods [10].

This study reviews the state of the art in FDM printing of composite materials.

\footnotetext{
${ }^{\mathrm{a}}$ Corresponding author : k.bryll@am.szczecin.pl
} 


\section{Fused deposition modeling (FDM)}

FDM technology also known as fused filament fabrication (FFF) is currently the most widespread way to rapid production of items utilizing additive manufacturing [1-5]. This method is a well-known technology patented in 1989 by Scott Crump, a co-founder of the Stratasys company [1].

3D printing by FDM consists in depositing a filament of thermoplastic material. A portion of filament from a reel passes through a hot head, with a temperature higher than the melting point of the filament, then is extruded in the XY plane creating a layer of solid material on the build plate.

Creating a model can be done by depositing a layer contour, then filling the inside with plasticized material by zigzag movement of the head. After printing one layer the head moves along the $\mathrm{Z}$ axis initiating the build-up of the next layer. Using this technique, we can create complex shapes with a minimum of preparatory processes. The production process begins with creating a model in the CAD program, then the model is incorporated into the program enabling control of process parameters such as head movement, feed rate, layer thickness, infill, head and table temperatures, slicing, support application, etc. Such a program generates a G-code, which uploaded in a 3D printer enables making a real model. The model removed from the printer needs finish machining, for instance to remove the supports and imperfections [2-9].

The diagram of 3D printing procedure by FDM method is shown in Fig. 1.

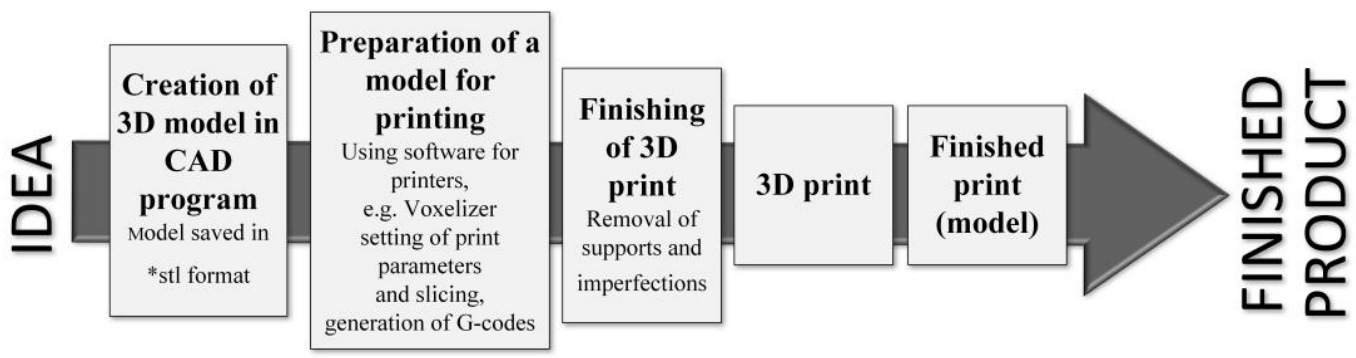

Figure. 1. Procedure of FDM printing

The most common drawbacks associated with FDM technology include [2-9]:

- stepped layers - visible paths of the deposited material due to a certain distance between edges of subsequent layers. This effect can be minimised by printing lower layers as well as reducing the diameter of the extruder (which will extend the process and raise the cost).

- overhang and bridging - printing of each model begins from the first layer applied onto a build plate. A subsequent layer is printed onto the surface of the previous one. If an item has elements set at an angle relative to the vertical axis (e.g. at $45^{\circ}$ ) the filament may not have a support and collapse. In such cases, supports should be generated at the model preparation stage to prevent damage. A bridge, that is parts of the model supported on its two ends, is a particular, troublesome case of overhang [9]. Therefore, in the case of long overhangs, auxiliary supports have to be designed, then removed during the final machining of the print. Alternatively, we can try to divide a model and place it on the build platform so that the bridges can be set upright.

- stringing (oozing) - occurs if during the extruder movement between two points some of the plasticized filament leaks down due to gravitation. In order to remove this effect, appropriate retraction should be set, a process of shifting the filament back into the nozzle.

- 'warping' of the model occurs when the edges or corners of the model rise above the table, deforming the model. The process takes place due to material shrinking and uneven temperature distribution across the model. We can partly counteract the process by changing temperature settings of the table, or putting a kapton tape on the table surface.

- hygroscopicity (term commonly used in works [2-7], although these authors propose to call this irregularity 'occluded or precipitated porosity'), is a property of polymer materials, leading to deteriorated print quality. Under the influence of nozzle temperature, moisture in the material leads to 
the creation of air bubbles in the model structure. This process can be minimised if filament is stored in appropriate conditions or dried before printing.

- 'structural inhomogeneity', a term proposed by the authors, means inequality, discontinuity, undulation, heterogeneity of structure particle size or insufficient density of the object, a characteristic of the manufacturing process. Structural inhomogeneity is connected with selective deposition of the filament layer by layer on the surface, by temperature differences, or porosity. This can be minimised by reducing the length between the spits of the filament.

- print damage by the printer head due to insufficient accuracy of nozzle movement.

\section{Methods of printing polymer composites using FDM technology}

Based on the review of publications [2-24] it is proposed to divide methods of printing composite materials or multimaterial structures by type of the extruder (printing head) Fig 2.

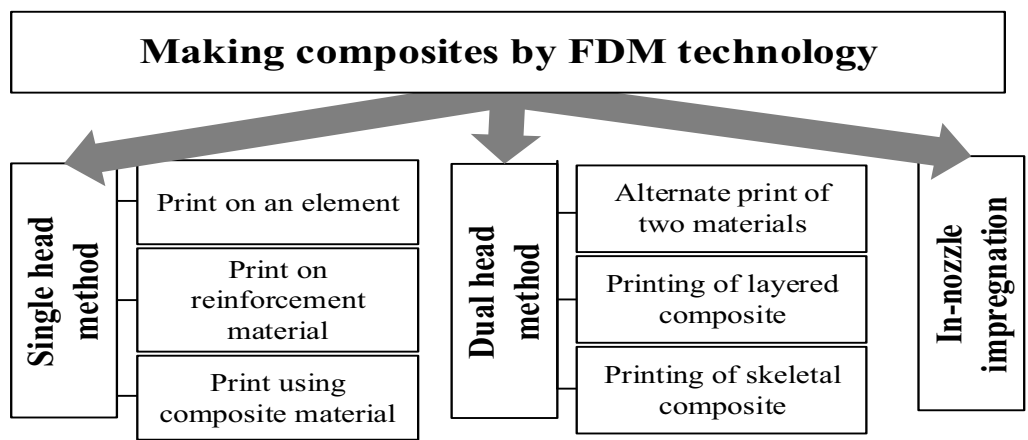

Figure 2. Composite manufacturing by FDM technology

\subsection{Creating multimaterial structures by printing on an element}

An interesting method of making multimaterial structures is printing a layer of polymer material on the surface of a ceramic, metal, wooden or polymer material. Structural elements obtained in this way may be composed of a few materials, in which the built-in element occupies much of the entire volume [5-6].

The biggest limitation of this method is the need to divide the model into parts separated by parallel planes, identifying subsequent printing stages. Besides, the printing head has to be controlled and stopped so that additional elements can be placed. The technological problem in this method is resuming printing without breaking the continuity of the material [5-6].

\subsection{Creating a composite structure by single nozzle printing on the reinforcement material}

Manufacturing a composite structure using a single head by printing on the reinforcement material (Fig 3a), is a method similar to creating multimaterial structures. Composite materials are obtained in a multi-stage process, where the number of stages depends on the number of reinforcement layers in a finished element. Each printing stage is designed by specifying a division plate, which enables stopping the printing and depositing the reinforcement phase on the print. Layered composites made by this method can be reinforced with continuous fibre, cloth, or mat [5]. 


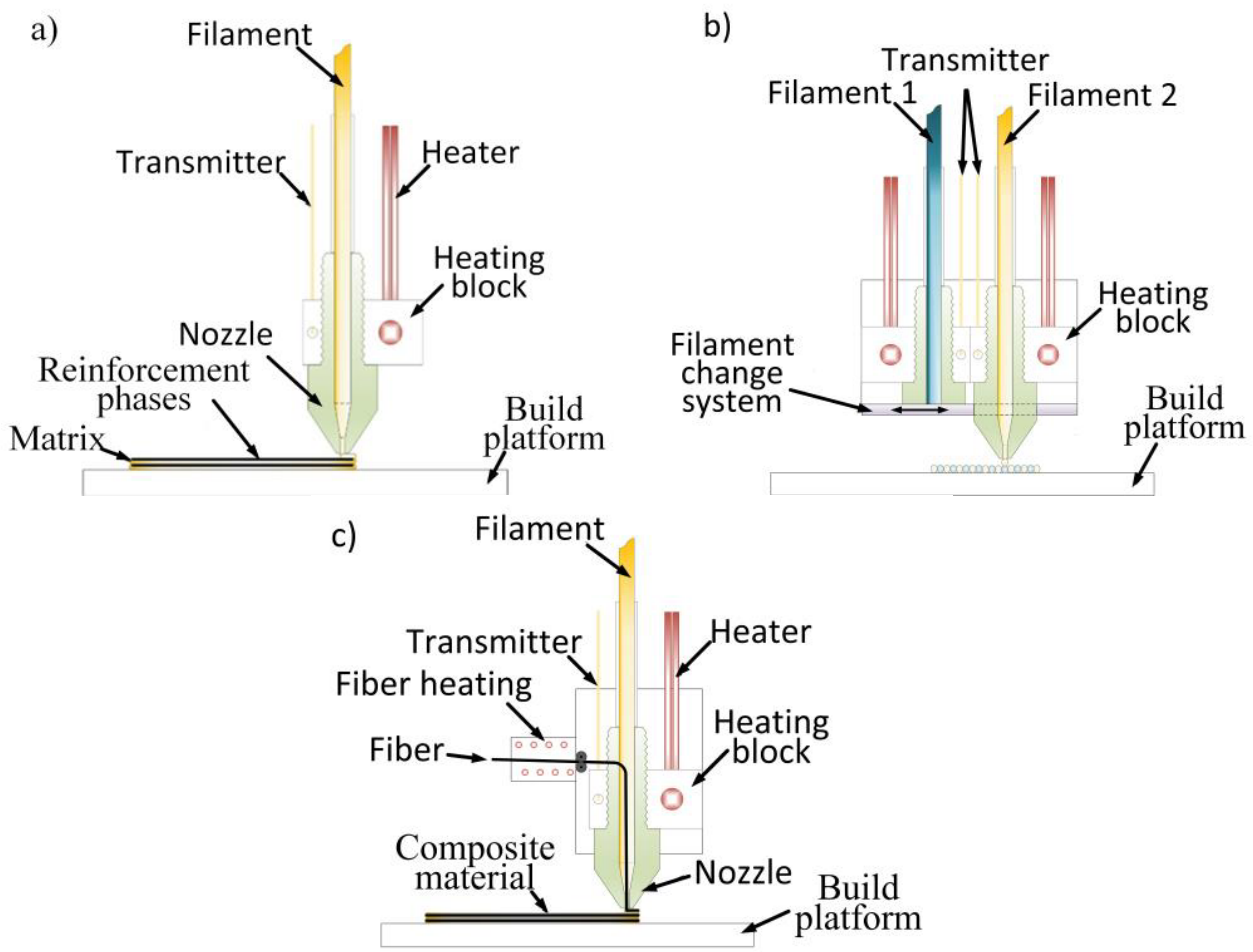

Figure. 3 Manufacturing and composite structure by:

a) single head printing on a reinforcement material

b) dual materials printing

c) in-nozzle impregnation

Disadvantages of this type of printing include restrictions associated with the process and with the thickness of individual layers of the reinforcement material in the composite. If a bunch of reinforcing fibres is too thick, the reinforcement phase may get deformed because fibers stuck to a jet of plasticized material may get shifted. The layer of reinforcement commonly found in the literature [2-4] is approximately $0.5 \mathrm{~mm}$ thick.

\subsection{Creating a composite structure with a single nozzle using a specific filament}

Manufacturing a composite structure with a single nozzle uses a different filament from that in traditional 3D printing technology; which besides polymeric material contains an additive: rubber microspheres, particles of glass or carbon fibre, wood flour etc.

The biggest limitation to this method is the availability of the material for printing. A prepared filament can be purchased, but currently the market offers a limited quantity of such products [4-6, 1013].

Composite filament could also be produced as a result of extruding a monofilament of defined diameter. However, it is a process requiring specialist equipment and know-how. For instance, to obtain the required filament diameter $(1.75 \mathrm{~mm}$ or $3 \mathrm{~mm})$, it should be extruded at a specific speed. The greater the speed, the smaller is the filament diameter. There are also limitations to the particle size and volumetric fraction of reinforcement in the filament. Excessive particle sizes and/or volume fraction may lead to blocking the flow of 3D printer nozzle, which will result in defective print [4-6, 10-13]. 


\subsection{Creating a composite structure by alternate dual material printing}

A diagram illustrating the making of a composite structure by means of alternate printing using two materials is given in Figure $3 \mathrm{~b}$.

The process of selective printing with two materials is an advanced 3D printing method, getting increasingly more common. It can be done using: a head consisting of two nozzles or newer solutions of one nozzle with a filament change system.

The method consists in alternate deposition of different materials in one printing process. The advantage of this method is the possibility to control the properties of a finished item at the design stage, because we can design and then make any composite structure (Fig. 4 a-b).

The greatest limitations of that manufacturing method stem from dual material printing imperfections, e.g. contamination due to the impossibility of transition from one filament to the other, fragment after fragment, as well as problems in proper configuring of the G-code, and the requirement of using materials with similar thermal characteristics. These problems are being constantly solved by introducing new systems of filament change and software updates by printer manufacturers.

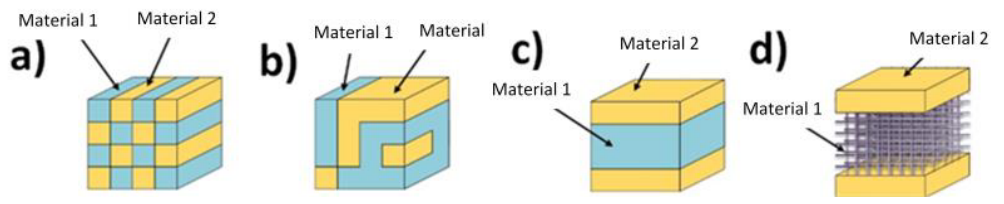

Figure. 4 Exemple print structures of dual materials composite

a-b) varied distribution of materials c) sandwich structured composite - layered, d) sandwich structured composite - skeletal.

\subsection{Creating a layered composite structure using two materials}

Creating a layered composite structure is the form of printing using two materials. An essential difference between these methods is the manner of material deposition, which in this case is layered, first material no 1, then material no 2, no 1 again etc. A schematic structure obtained by this method is shown in Fig. 4c.

\subsection{Creating a skeletal composite structure using two materials}

Printing a skeletal composite is similar to printing a layered composite, the biggest difference being that one of the materials is not solid, but a skeletal structure (Fig. 4d).

To produce such structures we can use algorithms reducing infills, known from printing prototypes technology, which have helped to save time and materials in the process of printing. The most common skeletal structures include hexagonal cells, square cells or honeycomb; other structures individually designed can be used as well, given that the FDM method has a limitation concerning overhangs known as bridges.

\subsection{Creating a layered composite structure using a special nozzle allowing introduction of the continuous fiber}

Figure $3 \mathrm{c}$ schematically shows the making of continuous composites by in-nozzle impregnation.

Filament being the matrix material and continuous reinforcing fibers are supplied separately to the printer nozzle. In order to increase fibre impregnation with thermoplastic material, before its insertion in the nozzle the reinforcement phase is heated. Fibers are automatically supplied to the head through filament motion. Filament is plasticized inside the printer head and connected with the reinforcement phase. Subsequent stages of the printing process are similar to traditional 3D printing. 


\section{Summary}

3D printing enables making single pieces of complex parts based on a CAD model without the use of expensive tools or moulds. The development of this technology results in prints in different areas of life. Of a few 3D printing systems, the one based on FDM modelling is particularly widespread thanks to the simplicity and capabilities of the method. Other advantages include prices of the printers dropping, better software and a broadening scope of materials used, including composites, improving mechanical properties of $3 \mathrm{D}$ prints.

The literature on the subject [2-22] contains numerous publications addressing 3D printing of composite materials. Various types of reinforcement can be used, for instance carbon structures [14,16-17] reinforcing plates [11] and short fibres: carbon [14], polymer [15] and glass [18].

However, the majority of research refers to the preparation and printing of composites from filament. Other methods using 3D printing with two heads are mentioned in few publications [7, 1819]. These composites usually show worse mechanical properties compared to composites produced by conventional methods, because composites reinforced with short fibres or particles are mechanically worse than those strengthened with continuous fibres [20-22]. That is why 3D printing using continuous fibres is an interesting solution [23-24]. 3D printed composites are designed to make elements of high electric conductivity [13,17], low coefficient of thermal expansion and high thermal conductivity [22].

Despite increased interest in printing composite materials and many publications on the developments of this technology, there are still numerous issues requiring further analysis. Essential technical problems to be solved are associated with intermittent printing or filament changes. It is also necessary to seek new material connections and adapt them for printing, provided that such solutions are cost-effective and environmentally acceptable.

This requirement may be satisfied by single polymer composites, in which both the matrix and reinforcement are made of the same or similar polymeric material, where components can vary in molecular weight, density or degree of branching [25].

Issues of 3D FDM printing of single polymer polyester composites will be discussed by these authors in subsequent publications.

\section{Acknowledgments}

The research presentedin this article was carried out under the Grant of the Ministry of Science and Higher Education of Poland nr 1/S/IESO/17 performed at the Maritime University of Szczecin, Poland.

\section{References}

1. Patent US 5121329 A: Apparatus and method for creating three-dimensional objects; 9 Cze 1992

2. Fuda Ning, Weilong Cong, Jingjing Qiu, Junhua Wei, Shiren Wang: Additive manufacturing of carbon fiber reinforced thermoplastic composites using fused deposition modeling; Composites Part B: Engineering;Volume 80, 2015, Pages 369-378

3. Nannan GuoMing C. Leu: Additive manufacturing: technology, applications and research needs; Frontiers of Mechanical Engineering; 2013, Volume 8, Issue 3, pp 215-243

4. Kumar S. \& Kruth J.-P. Composites by rapid prototyping technology. Materials \& Design 31, 850-856 (2010).

5. Dudek P.: FDM 3D printing technology in manufacturing composite elements. Archives of Metallurgy and Materials, 58(2013),nr.4,1415-1418

6. David Bak, "Rapid prototyping or rapid production? 3D printing processes move industry towards the latter", Assembly Automation, 2003, Vol. 23 Issue: 4, pp.340-345

7. Sung-Hoon AhnEmail authorKyung-Tae LeeHyung-Jung KimRenzhe WuJi-Soo KimSung-Hyuk Song Smart soft composite: An integrated 3D soft morphing structure using bend-twist coupling 
of anisotropic materials International Journal of Precision Engineering and Manufacturing; 2012, Volume 13, Issue 4, pp 631-634

8. Bakarich S. E., Gorkin R. 3rd, in het Panhuis M. \& Spinks G. M. Three-dimensional printing fiber reinforced hydrogel composites. ACS applied materials \& interfaces 6, 15998-16006 (2014). [PubMed]

9. Suwanprateeb J., Sanngam R., Suvannapruk W. \& Panyathanmaporn T. Mechanical and in vitro performance of apatite-wollastonite glass ceramic reinforced hydroxyapatite composite fabricated by 3D-printing. J Mater Sci Mater Med 20, 1281-1289 (2009). [PubMed]

10. Chybowski L., Gawdzińska K., On the Possibilities of Applying the AHP Method to a Multicriteria Component Importance Analysis of Complex Technical Objects. Advances in Intelligent Systems and Computing, Volume 445, Springer International Publishing 2016, pp. 701-710 [ISSN 2194-5357, ISBN 978-3-31306-1, online ISBN 978-3-319-31307-8, DOI 10.1007/978-3319-31307-8_71]

11. Dimas L. S. \& Buehler M. J. Modeling and additive manufacturing of bio-inspired composites with tunable fracture mechanical properties. Soft Matter 10, 4436-4442 (2014). [PubMed]

12. Compton B. G. \& Lewis J. A. 3D-printing of lightweight cellular composites. Adv Mater 26, 5930-5935 (2014). [PubMed]

13. Leigh S. J., Bradley R. J., Purssell C. P., Billson D. R. \& Hutchins D. A. A simple, low-cost conductive composite material for 3D printing of electronic sensors. PLoS One 7, e49365 (2012). [PMC free article] [PubMed]

14. Tekinalp H. L. et al. Highly oriented carbon fiber-polymer composites via additive manufacturing. Compos Sci Technol 105, 144-150 (2014).

15. Gray R. W. IV, Baird D. G. \& Bohn J. H. Thermoplastic composites reinforced with long fiber thermotropic liquid crystalline polymers for fused deposition modeling. Polym Compos 19, 383394 (1998).

16. Shofner M. L., Lozano K., Rodriguez-Macias F. J. \& Barrera E. V. Nanofiber-reinforced polymers prepared by fused deposition modeling. Journal of Applied Polymer Science 89, 30813090 (2003).

17. Pidcock G. C. \& in het Panhuis M. Extrusion Printing of Flexible Electrically Conducting Carbon Nanotube Networks. Advanced Functional Materials 22, 4790-4800 (2012).

18. Zhong W., Li F., Zhang Z., Song L. \& Li Z. Short fiber reinforced composites for fused deposition modeling. Materials Science and Engineering A 301, 125-130 (2001).

19. Scheithauer U., Bergner A., Schwarzer E., Richter H.-J. \& Moritz T. Studies on thermoplastic 3D printing of steel-zirconia composites. Journal of Materials Research 29, 1931-1940 (2014).

20. Hsueh C.-H. Young's modulus of unidirectional discontinous-fibre composites. Compos Sci Technol 60, 2671-2680 (2000).

21. Hine P. J., Lusti H. R. \& Gusev A. A. Numerical simulation of the effects of volume fraction, aspect ratio and fibre length distribution on the elastic and thermoelastic properties of short fibre composites. Compos Sci Technol 62, 1445-1453 (2002).

22. Love L. J. et al. The importance of carbon fiber to polymer additive manufacturing. Journal of Materials Research 29, 1893-1898 (2014).

23. Ryosuke Matsuzaki, Masahito Ueda, Masaki Namiki, Tae-Kun Jeong, Hirosuke Asahara, Keisuke Horiguchi, Taishi Nakamura, Akira Todoroki, and Yoshiyasu Hirano:Three-dimensional printing of continuous-fiber composites by in-nozzle impregnation; Sci Rep. 2016; 6: 23058.

24. Frank Van Der Klift, Yoichiro Koga, Akira Todoroki, Masahito Ueda, Yoshiyasu Hirano, Ryosuke Matsuzaki:3D Printing of Continuous Carbon Fibre Reinforced Thermo-Plastic (CFRTP) Tensile Test Specimens; Open Journal of Composite Materials, 2016, 6, 18-27

25. K. Bryll, K. Gawdzińska, M. Nabiałek, P. Pawłowska; The effect of degradation in aqueous media on viscosity average molecular weight of single polymer polyester composites; Revista de Chimie 2017; 68(9): 2034-2038. 